\title{
Central Stars of Young Planetary Nebulae - A New Class of Variables
}

\author{
G. Handler
}

South African Astronomical Observatory, P.O. Box 9, Observatory 7935, South Africa

\begin{abstract}
A new class of variable star is proposed. These are variable central stars of young Planetary Nebulae exhibiting roughly sinusoidal (semi)regular photometric and/or radial velocity variations with time scales of several hours. Fourteen of these objects have been identified. Their temperatures are between 25000 and $50000 \mathrm{~K}$ and most show hydrogen-rich spectra. The most likely reason for the variability is stellar pulsation. Another possibility would be variable stellar mass loss, but in that case the mechansism causing it must be different from that operating in massive $\mathrm{O}$ stars. We speculate that it actually is the stellar pulsations which cause mass loss mdulations.
\end{abstract}

\section{Introduction and observations}

In the late 1980s and early 1990 s, several variable central stars of planetary nebulae (CSPN) were noted which showed similar behaviour. They are all central stars of young PN $\left(25000 \mathrm{~K}<T_{\text {eff }}<50000 \mathrm{~K}\right)$ and no well-defined single periodicity was found in light and radial velocity curves. What is the reason for the variability? Variable mass loss? Pulsations?

In the latter case, the measurement of period changes (which would be enormous) would allow to determine masses and distances to the individual central stars, resolving a long-standing problem in PN research.

Consequently, we conducted extensive photometric obervations of these stars, including multisite time-series photometry of the central star of IC 418 (Handler et al. 1997), extensive time-series photometry of the central star of M2-54 (Handler 1999), a search for variability amongst Northern and Equatorial CSPN (Handler 1998), and the completion of this survey in the Southern Hemisphere (Handler 2002).

\section{Results and discussion}

We confirmed 4 known/suspected variables, detected 10 new ones, and have a number of less convincing cases for variability. The established variables (listed in Table 1) vary on two time scales: of the order of several hours and/or of the order of several days. The physical reasons for the variations on the different time scales may not be the same. 
Table 1. Established variable central stars of young Planetary Nebulae

\begin{tabular}{|l|c|c|c|c|}
\hline Central star of & Spectral type & V (mag) & Time scale (hr) & $T_{*}(\mathrm{kK})$ \\
\hline H 2-1 & O(H) & 13.25 & $>5$ & 33 \\
He 2-131 & Of(H) & 1.01 & $>9$ & 26 \\
He 2-138 & O(H) & 10.90 & $>9$ & 27 \\
He 2-151 & O(H) & 13.08 & $>9$ & 25 \\
IC 418 & Of(H) & 9.93 & $6.5 \pm 0.15$ & 36 \\
IC 2149 & Of(H) & 11.59 & $5.0 \pm 2.2$ & 42 \\
IC 4593 & O5f(H) & 11.20 & $>4.5$ & 40 \\
M1-46 & Of(H) & 12.83 & $>7$ & 45 \\
M1-77 & OB? & 12.12 & $>8$ & $<26$ \\
M2-54 & B? & 12.08 & 8.9 or 14.3 & 30 \\
NGC 2392 & Of(H) & 10.53 & $5.3 \pm 1.1$ & 47 \\
NGC 6543 & Of-WR(H) & 11.14 & $3.5 \pm 1.5$ & 45 \\
NGC 6826 & O3f(H) & 10.41 & $3.8 \pm 1.7$ & 50 \\
Tc 1 & Of(H) & 11.85 & $>6$ & 33 \\
\hline
\end{tabular}

The temperature range in which the photometric variations occur $(25000-$ $50000 \mathrm{~K}$ ) is in better agreement with the idea that the reason for the variability is due to stellar pulsation as opposed to wind variability, and the ratio of variables to constant stars in this temperature range is too large for the variations to be caused by binarity only. The time scales of the faster variations are clearly too short to be consistent with the hypothesis of rotationally induced wind variability, as believed to be present in massive O stars (e.g. Kaper et al. 1996). In addition, all CSPN exhibiting short-term variability are hydrogen-rich, suggesting the variations originate in the photosphere.

\section{Conclusions and suggestions}

We are in the presence of a new class of variable star. Their most important common characteristics are (other similarities are just trivial consequences of these points): they exhibit (roughly) sinusoidal (semi)regular photometric and/or radial velocity variations with time scales of several hours, they have effective temperatures less than $50000 \mathrm{~K}$, and all related variables so far have hydrogen-rich spectra

It is proposed that this new class of variables will henceforth be called $\mathrm{ZZ}$ Leporis stars, after its prototype, the central star of IC 418. Spectroscopists studying wind variations of CSPN are encouraged to examine the time scales on which these occur and to perform simultaneous photometry to constrain the cause of variability of these stars better.

\section{References}

Handler, G., et al. 1997, A\&A 320, 125

Handler, G., 1998, PhD thesis, University of Vienna

Handler, G., 1999, A\&AS 135, 493

Handler, G., 2002, in preparation

Kaper, L., et al., 1996, A\&AS 116, 257 